\title{
The Effect of Group Support Psychotherapy Delivered by Trained Lay Health Workers for Depression Treatment Among People with HIV in Uganda: Protocol of a Pragmatic, Cluster Randomized Trial
}

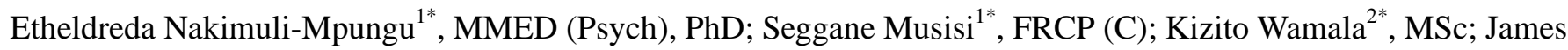
Okello $^{3^{*}}$, MMED (Psych), PhD; Sheila Ndyanabangi ${ }^{4 *}$ MBChB, MPH; Ramin Mojtabai ${ }^{5^{*}}$, MD, PhD; Jean Nachega $^{6,7,8,9 *}, \mathrm{MD}, \mathrm{PhD}$; Ofir Harari ${ }^{10^{*}}, \mathrm{PhD}$; Edward Mills ${ }^{11^{*}}, \mathrm{PhD}$

\footnotetext{
${ }^{1}$ Department of Psychiatry, College of Health Sciences, Makerere University, Kampala, Uganda

${ }^{2}$ Department of Psychology, Center for Victims of Torture, Gulu, Uganda

${ }^{3}$ Department of Mental Health, Faculty of Medicine, Gulu University, Gulu, Uganda

${ }^{4}$ Mental Health Program, Ministry of Health of Uganda, Kampala, Uganda

${ }^{5}$ Department of Mental Health, Bloomberg's School of Public Health, Johns Hopkins University, Baltimore, MD, United States

${ }^{6}$ Department of Epidemiology, Pittsburg Graduate School of Public Health, University of Pittsburg, Pittsburgh, PA, United States

${ }^{7}$ Stellenbosch Center for Infectious Disease, Department of Medicine, Stellenbosch University, Cape Town, South Africa

${ }^{8}$ Department of Epidemiology, Bloomberg's School of Public Health, Johns Hopkins University, Baltimore, MD, United States

${ }^{9}$ Department of International Health, Bloomberg's School of Public Health, Johns Hopkins University, Baltimore, MD, United States

${ }^{10}$ MTEK Sciences Inc, Vancouver, BC, Canada

${ }^{11}$ Department of Clinical Epidemiology \& Biostatistics, McMaster University, Hamilton, ON, Canada

*all authors contributed equally
}

\section{Corresponding Author:}

Etheldreda Nakimuli-Mpungu, MMED (Psych), PhD

Department of Psychiatry

College of Health Sciences

Makerere University

Old Mulago Hospital Complex

Mulago Hill Road

Kampala, 256

Uganda

Phone: 256788994050

Email: ethelmpungu@yahoo.com

\section{Abstract}

Background: There is limited information on the effectiveness of task shifting of mental health services in populations with HIV.

Objective: This trial aims to evaluate the effectiveness of group support psychotherapy delivered by trained lay health workers to persons living with HIV (PLWH) with depression in primary care.

Methods: Thirty eligible primary care health centers across three districts were randomly allocated to have their lay health workers trained to deliver group support psychotherapy (intervention arm) or group HIV education and treatment as usual (control arm) to PLWH with depression. Treated PLWH will be evaluated at baseline, after the end of treatment, and at 6-month intervals thereafter for 2 years. Primary outcomes will be the difference in follow-up proportions of participants with Mini International Neuropsychiatric Interview criteria for major depression and difference in follow-up function scores of participants in the intervention and control arms 6 months after the end of treatment. Secondary outcomes will include measures of self-esteem, posttraumatic stress symptoms, social support, stigma, adherence to antiretroviral therapy, viral load, and number of disability days, asset possession indices, and cost-effectiveness data. Primary and secondary outcomes as well as subgroup analyses will be conducted at the individual level using multilevel random effects regression analyses adjusting for clustering in health centers. 
A process evaluation using mixed methods to assess acceptability, feasibility, fidelity, causal mediating processes, and contextual influences in the trial will be conducted.

Results: The trial has been approved by the Makerere College of Health Sciences School of Health Sciences Research Ethics Committee, the AIDS Support Organization, and the Uganda National Council of Science and Technology. A data and safety monitoring board has been put in place to monitor trial progress. A total of 1140 persons living with HIV have been recruited to the trial. An analysis of baseline and 6-month data is in progress. The results of this trial will not only be presented at national and international conferences but also submitted for publication in peer-reviewed journals and as a report to the funding agencies.

Conclusions: This cluster randomized trial will provide critical evidence to support culturally sensitive group-based psychotherapy for depression treatment in sub-Saharan Africa. Process evaluation outcomes will provide contextual information that health care and public health stakeholders can use to guide implementation decisions for their particular setting.

Trial Registration: Pan African Clinical Trials Registry (PACTR): 201608001738234; http://www.pactr.org/ATMWeb/ appmanager/atm/atmregistry?dar=true\&tNo=PACTR201608001738234 (Archived by WebCite at http://www.webcitation.org/ 6vUAgAQlj)

(JMIR Res Protoc 2017;6(12):e250) doi: 10.2196/resprot.8925

\section{KEYWORDS}

cluster randomized trial; group support psychotherapy; lay health workers; depression; persons living with HIV/AIDS; Uganda

\section{Introduction}

In the fight against the HIV epidemic over the past three decades, one of the major barriers to universal access to health care needed by persons living with HIV (PLWH) has been a serious shortage of health workers to deliver these interventions [1]. The shortage of well-trained health workers to address the myriad biological, social, and psychological challenges of living with HIV poses a risk for suboptimal HIV treatment outcomes, particularly in sub-Saharan African countries where the burden of HIV and acquired immune deficiency syndrome (AIDS) is greatest [2]. For example, the lack of capacity to provide mental health care such as screening for depression in HIV care programs results in undetected and untreated depression, which impairs the ability to adhere to antiretroviral (ART) medications $[3,4]$. Affected individuals continue to struggle with poor health, social, and economic outcomes because depression impairs their ability to function in their families, at work, and in their communities $[5,6]$.

The World Health Organization (WHO) HIV treatment guidelines now recognize depression treatment as central to effective HIV treatment programs [7]. This, in turn, has resulted in the development and testing of a number of psychological interventions for depression that can be used in poor resource areas where the gap between the availability and need for mental health services [8-10], known as the "treatment gap," may be as high as 90\% [11]. Unfortunately, accessibility and sustainability of these interventions is impeded by the severe shortage of mental health professionals who can deliver them to affected individuals who need them [12].

Task-shifting approaches, whereby nonspecialist health workers in primary care and community settings are trained to deliver some of the mental health services that were provided solely by specialist mental health professionals, have been recommended by global mental health researchers and practitioners [13] as well as the WHO. The WHO recommends that these task-shifting approaches must use standardized and simplified interventions that can realistically be administered by less highly trained professional health care workers and nonprofessional community members [2]. However, studies describing the implementation of task-shifting approaches in delivery of psychological interventions for PLWH in sub-Saharan Africa are limited.

In a recent review of studies documenting the effectiveness of psychological interventions for PLWH in low- to middle-income countries, none of the studies that met criteria for inclusion in the review demonstrated the use of a task-shifting approach in the delivery of the intervention [14]. In a recent review of studies describing mental health training of health care workers in Africa, only three of the 37 studies reviewed described training of lay health workers (LHWs) [15]. There is a need for information on how a task-shifting approach can be used to make a psychological intervention for depression accessible and sustainable in low-resource settings.

The shifting of mental health related tasks from health professionals to LHWs has been repeatedly documented in non-HIV populations [16-19]. However, because mental health has not been integrated into HIV care in sub-Saharan Africa, little is known about the effectiveness of task shifting of mental health related services such as depression care. Also, identifying mediators and moderators of intervention response is a critical step in understanding the mediating causal factors as well as for whom and under which conditions an intervention is most beneficial [20]. This knowledge also has important implications for practice, as it can aid in tailoring and modifying interventions so as to be maximally effective for specific target populations.

To address this knowledge gap, we set out to conduct a cluster randomized trial to test the effectiveness of group support psychotherapy (GSP) delivered by trained LHWs. The recommended methodological approach to establish the efficacy of a newly developed therapy such as GSP is to first compare the therapy against a comparison group that omits the unique ingredients of the new therapy while possessing the common factors (eg, therapeutic alliance) in equal measure [21]. Thus, we compared the effects of GSP to those of group HIV education (GHE) delivered by trained LHWs on mild to moderate 
depression and functioning among PLWH attending primary care health centers in northern Uganda. We hypothesized that compared with the control arm, the proportion of participants that meets the Mini International Neuropsychiatric Interview (MINI) criteria for major depression will be lower and the function scores will be higher in the intervention arm 6 months after the end of treatment.

Secondarily, we aimed to compare the effects of GSP and GHE delivered by trained LHWs to PLWH presenting with mild to moderate depression in primary care on measures of self-esteem, posttraumatic stress symptoms, social support, stigma, adherence to ART, viral load, number of disability days, asset possession, poverty indices, and cost-effectiveness measures. We hypothesized that compared with PLWH receiving GHE, those receiving GSP will achieve a greater increase in social support and positive coping skills and greater reduction in stigma; larger increases in adherence to ART and greater reduction in viral load; and greater reduction in number of disability days, greater increase in asset possession scores, and larger reductions in poverty index scores at $6,12,18$, and 24 months after the end of treatment.

We also plan to conduct a process evaluation of trial activities informed by Linnan and Steckler's process evaluation frameworks [22] to specifically determine indicators of feasibility including reach (ie, the proportion of participants who received the intervention), dose delivered and received (ie, the amount of intervention delivered and the extent to which participants respond to it), as well as attrition (ie, the proportion of participants who are lost to follow-up); indicators of acceptability including satisfaction with intervention content, delivery agents and effects; fidelity (ie, whether the intervention is delivered as planned); and causal mediating processes and contextual influences. Given that gender has far-reaching implications for social roles, opportunities, and experience of adversities in traditional societies such as in Uganda [23], we will determine whether or not the effects of GSP are moderated by gender. Both posttraumatic stress [24] and alcohol use disorders [25] are associated with an increased risk for depression. Therefore, we will also examine whether or not the effects of GSP are moderated by psychiatric comorbidities. Extensive reviews of literature [26,27] have found that common factors such as the therapeutic relationship may account for up to nine times greater impact on patient improvement than the specific mechanisms of action found in formal treatment protocols. Therefore, we shall assess whether the strength of a therapeutic relationship will mediate the effects of GSP on depression and subsequently other study outcomes. Identifying groups of individuals for whom GSP works best will assist in the goal of developing selection criteria to guide the referral of patients for GSP.

\section{Methods}

\section{Study Design}

This is a pragmatic two-arm cluster randomized trial evaluating the effectiveness GSP delivered by trained LHWs to persons with HIV presenting with mild to moderate depression in primary care. The study involves LHWs affiliated to 30 primary care health centers (PHCs) in three districts in northern Uganda. Eligible PHCs were randomly assigned (1:1) to have their LHWs trained in the delivery of GSP (intervention) or GHE (control) to PLWH with mild to moderate depression. PLWH treated by trained LHWs will be evaluated at baseline, at the end of intervention, and at intervals of 6 months thereafter for 2 years.

A longitudinal process evaluation of the delivery of GSP by trained LHWs using mixed methods will run alongside the trial to assess acceptability, feasibility, fidelity, and how intervention recipients respond to the different intervention components. Analysis is performed according to the intention-to-treat (ITT) principle and will account for the cluster randomized design. The study protocol is registered in the Pan African Clinical Trials Registry (PACTR201608001738234), and the reporting of the trial will be in accordance with the Standard Protocol Items: Recommendations for Interventional Trials (SPIRIT) guidelines [28] for intervention trials (see Multimedia Appendix 1 ) and the CONSORT statements for cluster randomized trials [29].

The study was submitted to and approved by both the Makerere University College of Health Sciences Research Ethics Committee and the Uganda National Council of Science and Technology. All study participants will be required to provide written informed consent. Light refreshments will be served during all group sessions in both arms, and every participant will receive a financial incentive to defray transport costs. Figure 1 summarizes the trial profile.

\section{Study Setting}

This trial has been implemented in 30 PHCs situated in three districts in northern Uganda (Gulu, Kitgum, and Pader) with a population of 450,000,247,000, and 250,000, respectively. Over $90 \%$ of the population is engaged in small scale agriculture and animal husbandry as their major income-generating activity. The brutal civil war that the people of these districts endured for two decades (1987-2007) led to a breakdown of health care delivery systems, disorganized social and cultural life with minimal economic activity, and loss of property and infrastructure in these districts. This situation of precariousness led to high poverty rates, risky behaviors including excessive alcohol consumption, and sexual violence making the population highly vulnerable to depression and HIV/AIDS [30].

The first level of health care accessible to this study population is provided by LHWs who work in teams referred to as village health teams. The village health team is affiliated with other PHCs at the second level (Health Center II), third level (Health Center III), or fourth level (Health Center IV), which are led by professional health workers who supervise their outreach activities [31]. 
Figure 1. Trial flowchart: selection of clusters and participants.

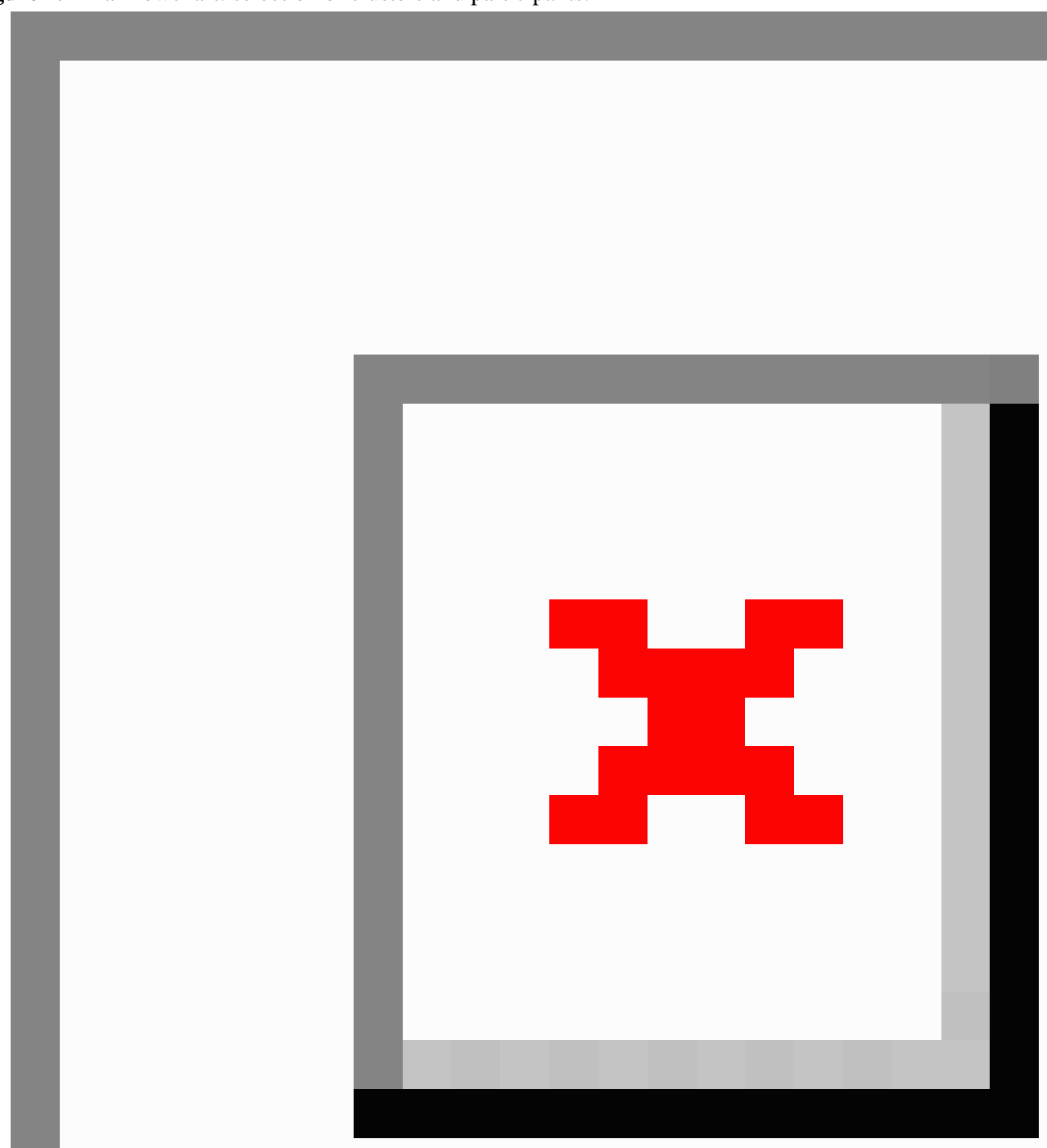

\section{Participants}

\section{Cluster Eligibility Criteria, Recruitment, and Randomization}

To be eligible for the trial, the primary care health centers had to offer HIV care and treatment services, nominate at least four LHWs (2 men and 2 women) who are actively involved in HIV care, able to read and write, and reside within the villages served by the PHC. PHCs that do not offer HIV care services and those unable to nominate literate LHWs were excluded from the study.

To recruit PHCs, the project team worked with the district health officials in each of the three participating districts and identified a total of 38 PHCs that offered HIV care and treatment services.
The health center managers of these centers were informed about the trial and asked to nominate health workers to participate in the trial. The first 10 PHCs in each district that expressed interest in the trial and nominated the required number of health workers were recruited to the trial. The health center managers of the 10 eligible PHCs in each district were invited to a stakeholders' meeting held at the district's local government offices with political leaders, district health officials, religious leaders, and community leaders. Study purpose and procedures were explained to facilitate district leadership understanding of the trial activities. At the end of the meeting, the eligible PHCs were randomly allocated with a 1:1 ratio to intervention and control arms. Randomization was done by urn randomization 
picked by each health center manager or their representative who attended the stakeholders' meeting.

\section{Participant Eligibility Criteria, Recruitment, and Masking}

To be eligible for the study, PLWH had to be 19 years and older, diagnosed with major depression assessed with the MINI depression module [32], antidepressant naïve, using ART, and residing in the villages where the trained LHWs lived. Individuals with high suicide risk [33], a severe medical disorder such as pneumonia or active tuberculosis, psychotic symptoms, and hearing or visual impairment were excluded from the study.

On a given clinic day, project research assistants worked with primary care health workers of a participating $\mathrm{PHC}$ in the center's HIV clinic. The primary care health worker gave a health talk on depression to clients in the waiting area. Clients who felt that they had experienced symptoms of depression described in the health talk were invited for further evaluations using the Luo version of the 20-item self-reporting questionnaire [34] and the MINI depression module. This procedure was repeated until a total of $40 \mathrm{PLWH}$ diagnosed with major depression were obtained from a given PHC.

Clients diagnosed with major depression were approached by research assistants who explained study procedures, determined eligibility, and then obtained informed consent. Each client who gave informed consent received baseline assessments with a standardized questionnaire. Recruited participants from the same village were assigned to a trained LHW residing in or near their village to receive the intervention they had been trained to deliver (ie, either GSP or GHE).

By design, both experimental and control interventions were identifiable to participants but will be masked to outcome assessors, the Data and Safety Monitoring Board, and data analysts.

\section{Interventions}

The development of the GSP and GHE interventions has been described in detail in previous publications $[35,36]$. Multimedia Appendix 2 shows a detailed description of the content of both interventions, which has also been previously published [37].

\section{Group Support Psychotherapy Training}

Over a 4-month period (January-April 2016), Makerere University in collaboration with the Ministry of Health designed a GSP training program that consists of both formal and informal training. Between May and August 2016, the training-of-trainers model was used to deliver the training, whereby mental health specialists trained PHC health workers who in turn trained the LHWs. Formal training consisted of 8 training modules delivered in a 5-day training workshop that employed active learning techniques including role plays, brainstorming sessions, and small group discussions.

In brief, the first three modules including an overview of the training program, introduction to the GSP model, and introduction to depression and HIV/AIDS were delivered on the first day. On the second and third days, modules on basic counseling skills and effective coping strategies were delivered, respectively. On the fourth day, participants received training in basic livelihood skills (enterprise selection, basic financial skills, and resource mobilization) required to overcome poverty. The last day of training focused on self-care strategies, post-training assessments, and training workshop evaluation. Informal training consisted of conducting supervised pilot GSP sessions. Multimedia Appendix 3 summarizes the competencies targeted by the training.

\section{Group HIV Education Training}

In May 2016, Makerere University in collaboration with The AIDS Support Organization (TASO), designed a group HIV education training program that consisted of both formal and informal training. Between May and August 2016, the training-of-trainers model was used to deliver the training, whereby TASO HIV care providers trained PHC health workers who in turn trained the LHWs. Formal training consisted of five training modules delivered in a lecture format in a 2-day training workshop. In brief, on the first day, three modules including an overview of the training program, introduction to depression and HIV/AIDS, HIV progression and transmission were delivered. On the second day, modules on mother-to-child transmission and basic facts on ART were delivered. Informal training consisted of conducting supervised pilot GHE sessions.

\section{Intervention Fidelity}

Between September and December 2016, trained LHWs delivered the interventions to PLWH with mild to moderate depression recruited to participate in the trial. Strategies to ensure treatment fidelity in both treatment arms included the use of standardized intervention materials, structured health worker training, ongoing supervision, and training a larger number of LHWs than was required in order to avoid potential disruptions due to illness or job transfers. The LHWs underwent standardized training by trained primary care health workers. Each LHW delivered GSP or GHE following a manual translated into the local language. Prior to the sessions, each LHW drew up a schedule indicating which day of the week each group session would take place and handed it over to their supervisor. Of the 8 sessions to be delivered, 3-4 sessions were supervised by a trained PHC worker who assessed the LHW's basic counseling skills using a supervision checklist with items adapted from the Enhancing Assessment of Common Therapeutic Factors scale [38].

In addition, the LHWs who facilitated group sessions were required to complete a self-administered semistructured feedback questionnaire after each group session in which they described content delivered in each group session and provided feedback on facilitators and barriers faced during the group session. Their feedback was discussed with their supervisors who advised on how to address any difficult issues raised. From this feedback, supervisors were able to assess whether the intervention delivery progressed as planned or not and to intervene accordingly.

\section{Participant Safety}

During baseline assessments, participants were carefully screened and individuals for whom the interventions were deemed medically inappropriate or unsafe were excluded. 
Outcome assessors screened all participants for adverse events at the end of treatment and at 6 months after the end of the intervention using a standard interview and reporting form. Going forward, follow-up assessments will continue at 6-month intervals up to 2 years after the end of treatment. Any unfavorable and unintended sign or symptom associated with the participation in either intervention, regardless of whether it is considered related to the therapy is reviewed by the investigating team for seriousness, study relatedness, and expectedness. Similar information reported by participants at other times (eg, during intervention encounters) is duly noted and followed up with, as needed, to assure participant safety. Adverse events are reported according to the data and safety monitoring plan shown in Multimedia Appendix 4.

\section{Retention}

Currently, trial participants have received their 6-month outcome assessment and will be due for their 12-month outcome assessment in January-February 2018. In order to maximize adherence and retention, we formed community advisory boards to monitor community satisfaction with implementation activities and to provide feedback to investigators in real time on any conflicts or dissatisfaction arising from project implementation activities. Lay health workers who facilitated the group sessions and the primary care health workers who supervised them were provided with a financial incentive as an appreciation of their commitment to the project. For participants who missed group sessions, the LHWs made home visits to re-engage them. Given that group sessions took place in the villages, the LHWs know the homes of the participants and have been able to mobilize them to return for their follow-up assessments.

\section{Study Measures and Data Collection Schedule}

So far, assessments of study measures have been conducted at baseline, at the end of the interventions ( 2 months), and 6 months after the interventions. Going forward, further assessments will be conducted at 12, 18, and 24 months after the intervention. Table 1 [32,34,39-47] summarizes the study measures including the primary and secondary outcomes, process evaluation outcomes, potential effect modifiers, and mediators. 
Table 1. List of study measures and data collection schedule.

\begin{tabular}{lll}
\hline Study measures & Instrument & Data collection schedule (months)
\end{tabular}

Primary outcomes

Major depression

Functioning level

\section{Secondary outcomes}

Depression symptoms

Posttraumatic stress symptoms

Alcohol use

Disability days

Cost-effectiveness

Poverty index scores

Adherence to ART

Viral load

\section{Potential effect modifiers}

Sociodemographic variables

Trauma events

\section{Potential effect mediators}

Self-esteem

Social support

HIV-related stigma

Coping skills

The therapeutic relationship

\section{Process evaluation outcomes}

Indicators of feasibility

Indicators of acceptability

Fidelity

Contextual influences

Causal mechanisms
The Mini International Neuropsychiatric Interview [32]

5-item locally developed function assessment method [39]

Self-Reporting Questionnaire [34]

Locally adapted Harvard Trauma Questionnaire [40]

10-item Alcohol Use Disorders Identification Test [44]

Single question "How many working days have you lost due to depression-related symptoms in the previous 30 days?

Estimation of incremental costs of the GSP intervention arm as compared to the control arm

Questions on indicators of socioeconomic status such as the building materials of the household dwelling unit, access to electricity, type of cooking fuel, source of lighting, household remoteness (distance to nearest health facility), household food security, household durable assets such as radios, televisions, and mobile phones

One question: "During the past week, on how many days have you missed taking all your medication doses?"

Medical charts of study participants

Standardized Demographic Questionnaire

Locally developed 16-item trauma event checklist

\section{Rosenberg Self-Esteem Scale [41]}

12-item Multi-Dimensional Perceived Social Support scale [42]

HIV-related stigma scale [43]

COPE Inventory [45]

The Scale to Assess the Therapeutic Relationships-Patient version [46]

The proportion of eligible participants who take up either intervention (Reach), the proportion who attended all 8 sessions of either intervention (dose delivered), and the proportion who are lost to follow-up (attrition) will be determined from the attendance registers

A 9-item questionnaire [47] will assess participant's satisfaction, the group facilitators' knowledge and attitudes, and the participant evaluation of the intervention's ability to reduce depression.

A semistructured self-administered questionnaire completed by group facilitators will assess whether or not the interventions were delivered as planned.

A semistructured self-administered questionnaire completed by group facilitators will assess any facilitators or barriers to intervention delivery that they observed during group sessions.

A semistructured interviewer-administered questionnaire will assess participant's knowledge, skills, or assets acquired during and after the interventions, which will give an indication as to whether or not the interventions influenced targeted risk factors for depression. 
Table 2. Sample size and power calculations.

\begin{tabular}{|c|c|c|c|c|c|c|c|}
\hline Alpha & Beta & $k$ & Cluster size & $\begin{array}{l}\text { Control group } \\
\text { proportion }\end{array}$ & $\begin{array}{l}\text { Intervention group } \\
\text { proportion }\end{array}$ & $\begin{array}{l}\text { Minimum clusters } \\
\text { required }\end{array}$ & $\begin{array}{l}\text { Resulting } \\
\text { power }\end{array}$ \\
\hline .01 & .2 & .25 & 32 & 0.25 & 0.15 & 18 & 0.808 \\
\hline .05 & .2 & .25 & 32 & 0.25 & 0.15 & 12 & 0.803 \\
\hline .01 & .2 & .25 & 40 & 0.25 & 0.15 & 16 & 0.818 \\
\hline .05 & .2 & .25 & 40 & 0.25 & 0.15 & 11 & 0.823 \\
\hline .01 & .2 & .25 & 32 & 0.3 & 0.15 & 10 & 0.842 \\
\hline .05 & .2 & .25 & 32 & 0.3 & 0.15 & 7 & 0.849 \\
\hline .01 & .2 & .25 & 40 & 0.3 & 0.15 & 9 & 0.851 \\
\hline .05 & .2 & .25 & 40 & 0.3 & 0.15 & 6 & 0.84 \\
\hline
\end{tabular}

\section{Sample Size and Power Calculations}

Primarily, our objective is to compare the proportion of subjects with mild to moderate depression in the intervention and control arms 6 months after the end of treatment. Based on results from our pilot project, we assume that the difference in proportion of depression cases at 6 months follow-up between intervention (15\%) and control arms (25\%) would be $10 \%$. Using formulae proposed by Hayes and Moulton [48], and assuming the between-cluster coefficient of variation $k$ of .25 , a study with 12 nonmatched pairs of clusters, and a cluster size of 32 PLWH (total expected sample size of 768 PLWH) would have $80 \%$ power of detecting a $10 \%$ reduction in major depression cases at the 5\% significance level. Table 2 illustrates the sample size and power calculations for various assumptions made. The number of clusters has been increased to 15 pairs to allow for individual level analyses using multilevel random effects regression models, and the cluster size was increased to 40 , accounting for a potential $20 \%$ loss to follow-up.

\section{Data Collection and Management}

Study participants in the intervention and control arms will be asked to complete an interviewer-administered face-to-face standardized questionnaire to collect data on primary and secondary outcomes at baseline (T0), after completion of their group treatments (T1), and 6, 12, 18, and 24 months after end of treatment (T2, T3, T4, and T5 respectively). All the completed survey questionnaires will be reviewed by a research team member for missing data and unusual responses. Data will be entered into an EpiData (version 3.1) database. Regular reports will be produced on (1) patient accrual and follow-up completion/retention in relation to goals and timeline, (2) the randomization process and group comparability on the balancing variables, (3) key baseline characteristics of the sample, by (blinded) group, related to the primary and secondary outcome variables and proposed effect modifiers and mediators, (4) intervention exposure and adherence, and (5) protocol violations. Any observed delays in these processes or data irregularities will be followed up and resolved in a timely manner.

The complete de-identified dataset of the trial will be publicly available when finalized. Details of the data and safety monitoring plan and the study governance structure are shown in the Multimedia Appendix 4.

\section{Statistical Analyses}

We will assess randomization across the two arms by comparing sociodemographic characteristics and other potential confounding variables using chi-square for categorical variables, and $t$ tests or other equivalent nonparametric tests, as appropriate, for continuous variables. Primary outcomes will be the difference in follow-up proportions of participants who meet MINI criteria for major depression and difference in follow-up function scores of participants in the intervention and control arms 6 months after the end of treatment.

Secondary outcomes will include measures of self-esteem, posttraumatic stress symptoms, social support, stigma, alcohol use, ART adherence, viral load, number of disability days, asset possession indices, and cost-effectiveness data. Primary outcomes, secondary outcomes, and all subgroup analyses will be analyzed by ITT using a multilevel mixed model adjusting for clustering in health centers to determine effect modifiers [49]. The status of randomization (intervention/control) and baseline depression score will be included as a covariate. To account for the clustered design, we will treat clusters as a random effect. Potential confounding variables (eg, age, gender, socioeconomic status) with significant differences across intervention and control arms will be included as fixed factors. The R statistical software will be used to conduct all analyses [50]. A process evaluation using mixed methods to assess acceptability, feasibility, fidelity, causal mediating processes, and contextual influences in the trial will be conducted.

The primary analysis will follow ITT principles and use all available follow-up data, with missing data handled directly through maximum likelihood estimation in mixed modeling. We will document the extent and pattern of missing data and the reasons and will conduct sensitivity analyses of the impact of missing data on stability of the primary results. Missing values will be imputed with multiple imputations. We will verify that mixed model based results are not sensitive to violations of model assumptions with permutation tests.

Last, Cohen $d$ effect sizes will be computed for the effect estimates to determine the size of the intervention effect [51].

For the qualitative data, interview transcripts will be reviewed for accuracy and then transcribed verbatim before translation into English. To control for errors in translation, two research assistants fluent in English and the local language (Luo) will 
work together to translate and electronically transcribe the data [52]. QRS NVivo 10 qualitative data analysis software will be used for coding and thematic analysis [53].

The interview data will initially be coded according to a number of themes that corresponded to the focus questions. The codes will be used to construct matrix displays based on the co-occurrence of codes and the two treatment groups. The resulting matrix display will provide both the frequency of responses and the detailed content of responses, allowing us to assess how often responses varied between the two treatment groups. Intercoder reliability will be assessed.

\section{Results}

The trial has been approved by the Makerere College of Health Sciences School of Health Sciences Research Ethics Committee, TASO, and the Uganda National Council of Science and Technology. A Data and Safety Monitoring Board has been put in place to monitor trial progress. A total of 1140 persons living with HIV have been recruited to the trial. An analysis of baseline and 6-month data is in progress.

The results of this trial will not only be presented at national and international conferences but also submitted for publication in peer-reviewed journals and as a report to the funding agencies.

\section{Discussion}

\section{Principle Findings}

The GSP intervention is a complex intervention containing several interacting components, requires those delivering the intervention to acquire certain competencies and skills, and is associated with a variety of outcomes. Its development was prompted by the need to narrow the gap between the availability and need for depression treatment among HIV-positive populations in poor resource settings. The prior feasibility study [54] and pilot randomized clinical trial [36] of this intervention served to test these theories, procedures, recruitment, retention, and explore our hypothesized change processes and outcomes. The process evaluation data of this trial indicated that acquisition of knowledge and skills that enhance social connections, support, and better coping strategies with adverse situations leads to a reduction in depression symptoms. The absence of depression improves ability to work and obtain savings and other livelihood assets. The pursuit of livelihoods helps restore the dignity and independence that sustain a reduction in depression and improvement in functioning [37].

The Social, Emotional, and Economic empowerment through Knowledge of Group Support Psychotherapy (SEEK-GSP) trial will provide robust evidence for the change processes and outcomes we observed in the pilot studies. Further, the trial will demonstrate the potential for dissemination and integration into existing HIV service delivery platforms of a culturally sensitive first line treatment for mild to moderate depression. Depression poses a major burden on persons living with HIV/AIDS, particularly those in poor resource settings where poverty and mental health interact in a negative cycle [55]. Treating depression in PLWH is critical to the realization of the
“90-90-90 targets" by 2020 aiming to diagnose $90 \%$ of all HIV positive people, provide ART for $90 \%$ of those diagnosed, and achieve viral suppression for $90 \%$ of those treated [56].

Prior trials of psychological therapies for depression in poor resource settings have shown that they result in significant reduction in depression symptoms and an increase in functioning levels $[57,58]$. However, both trials attracted mostly women. The SEEK-GSP trial specifically addresses this gap in evidence and in mental health services for a highly vulnerable HIV population in a primary care setting.

The SEEK-GSP trial targets both men and women living with HIV who have been exposed to decades of brutal civil war. Many of these individuals live in extreme poverty, with little to no education, in remote rural villages and rely on subsistence farming for survival. The vast majority have multiple experiences of war-related trauma and/or gender-based violence. This target group is a good example of what has been termed "intersecting populations"-groups that are vulnerable on multiple levels and disadvantaged across many determinants of health [59]. In this case, war survivors are made even more vulnerable by HIV/AIDS, unemployment, poverty, and food insecurity. Those living in remote villages with little access to services are even more vulnerable. This trial will demonstrate the extent to which primary care providers' influence can be leveraged to motivate affected individuals to attend group sessions, manage issues that arise during engagement, and support practice of learned skills. Such primary care based interventions also provide health care system opportunities to support culturally appropriate services that minimize unintended negative impacts and maximize positive impacts for vulnerable groups.

\section{Limitations}

Limitations of the SEEK-GSP trial relate to generalizability to other ethnic populations in Uganda. Also, the adherence measurement methods used in this study have limitations. However, to date there is no established gold standard to measure ART adherence. In the analyses of data from this trial, we shall assess the sensitivity of our adherence measure by determining whether it was able to predict viral suppression.

Despite these limitations, the trial will provide critical evidence to support culturally centered psychological therapy for depression in primary care settings. Trial outcomes will be supplemented with process evaluation outcomes that will provide contextual information that health care and public health stakeholders can use to guide implementation decisions for their particular setting [60].

\section{Conclusions}

Confirmation of our primary hypothesis and supportive secondary data can critically inform the national dissemination and implementation of GSP to treat mild to moderate depression in PLWH. Results may provide greater insight into the partnerships between LHWs and existing government health systems in low-resource settings and may lead to policy formulation regarding the integration of GSP into HIV care services on a country-wide basis. 


\section{Acknowledgments}

This study was funded by Grand Challenges Canada, Grant \#0770-05. EN-M is supported by the MQ Mental Health Fellowship Award, Grant \#MQ15FIP100024. The funders of the study had no role in study design, data collection, data analysis, data interpretation, or writing of the report.

\section{Authors' Contributions}

EN-M, KW, JO, SM, EJM, and JBN conceptualized and designed the study protocol and EN-M sought and obtained funding. $\mathrm{OH}$ conducted statistical analyses. EN-M and JO managed the literature searches. EN-M, JO, EJM, and JBN wrote the study protocol. SM, RM, EJM, OH, and JBN revised the protocol critically for important intellectual content. All authors contributed to the final study protocol.

\section{Conflicts of Interest}

None declared.

\section{Multimedia Appendix 1}

SPIRIT 2013 Checklist.

[PDF File (Adobe PDF File), 173KB-Multimedia Appendix 1]

\section{Multimedia Appendix 2}

The content of group support psychotherapy and group HIV education group sessions.

[PDF File (Adobe PDF File), 27KB-Multimedia Appendix 2]

\section{Multimedia Appendix 3}

Group support psychotherapy core competencies and subcompetencies.

[PDF File (Adobe PDF File), 32KB-Multimedia Appendix 3]

\section{Multimedia Appendix 4}

Data safety and management plan.

[PDF File (Adobe PDF File), 66KB-Multimedia Appendix 4]

\section{References}

1. Callaghan M, Ford N, Schneider H. A systematic review of task- shifting for HIV treatment and care in Africa. Hum Resour Health 2010 Mar 31;8:8 [FREE Full text] [doi: 10.1186/1478-4491-8-8] [Medline: 20356363]

2. World Health Organization. Task shifting: Global recommendations and guidelines. URL: http://www.who.int/healthsystems/ task shifting/en/ [accessed 2017-10-19] [WebCite Cache ID 6uKMNScEY]

3. Nakimuli-Mpungu E, Bass J, Alexandre P, Mills E, Musisi S, Ram M, et al. Depression, alcohol use and adherence to antiretroviral therapy in sub-Saharan Africa: a systematic review. AIDS Behav 2012 Nov;16(8):2101-2118. [doi: 10.1007/s10461-011-0087-8] [Medline: 22116638]

4. Uthman O, Magidson J, Safren S, Nachega J. Depression and adherence to antiretroviral therapy in low-, middle- and high-income countries: a systematic review and meta-analysis. Curr HIV/AIDS Rep 2014 Sep;11(3):291-307 [FREE Full text] [doi: 10.1007/s11904-014-0220-1] [Medline: 25038748]

5. Fried EI, Nesse RM. The impact of individual depressive symptoms on impairment of psychosocial functioning. PLoS One 2014;9(2):e90311 [FREE Full text] [doi: 10.1371/journal.pone.0090311] [Medline: 24587318]

6. Hamad R, Fernald LCH, Karlan DS, Zinman J. Social and economic correlates of depressive symptoms and perceived stress in South African adults. J Epidemiol Community Health 2008 Jun;62(6):538-544. [doi: 10.1136/jech.2007.066191] [Medline: 18477753 ]

7. World Health Organization. Consolidated guidelines on the use of antiretroviral drugs for treating and preventing HIV infection: recommendations for a public health approach. Geneva, Switzerland: World Health Organization; 2016. URL: http://www.who.int/hiv/pub/arv/arv-2016/en/ [accessed 2017-12-04] [WebCite Cache ID 6vTEIHVJX]

8. Nakimuli-Mpungu E, Wamala K, Okello J, Alderman S, Odokonyero R, Musisi S, et al. Outcomes, feasibility and acceptability of a group support psychotherapeutic intervention for depressed HIV-affected Ugandan adults: a pilot study. J Affect Disord 2014 Sep;166:144-150. [doi: 10.1016/j.jad.2014.05.005] [Medline: 25012423] 
9. Petersen I, Hanass HJ, Bhana A, Govender K. A group-based counselling intervention for depression comorbid with HIV/AIDS using a task shifting approach in South Africa: a randomized controlled pilot study. J Affect Disord 2014 Apr;158:78-84. [doi: 10.1016/j.jad.2014.02.013] [Medline: 24655769]

10. Chibanda D, Mesu P, Kajawu L, Cowan F, Araya R, Abas M. Problem-solving therapy for depression and common mental disorders in Zimbabwe: piloting a task-shifting primary mental health care intervention in a population with a high prevalence of people living with HIV. BMC Public Health 2011 Oct 26;11:828 [FREE Full text] [doi: 10.1186/1471-2458-11-828] [Medline: 22029430]

11. Kakuma R, Minas H, van Ginneken N, Dal PMR, Desiraju K, Morris JE, et al. Human resources for mental health care: current situation and strategies for action. Lancet 2011 Nov 05;378(9803):1654-1663. [doi: 10.1016/S0140-6736(11)61093-3] [Medline: 22008420]

12. Fulton B, Scheffler R, Sparkes S, Auh E, Vujicic M, Soucat A. Health workforce skill mix and task shifting in low income countries: a review of recent evidence. Hum Resour Health 2011 Jan 11;9:1 [FREE Full text] [doi: 10.1186/1478-4491-9-1] [Medline: 21223546]

13. Patel V, Collins PY, Copeland J, Kakuma R, Katontoka S, Lamichhane J, et al. The movement for global mental health. Br J Psychiatry 2011 Feb;198(2):88-90 [FREE Full text] [doi: 10.1192/bjp.bp.109.074518] [Medline: 21282777]

14. Chibanda D, Cowan FM, Healy JL, Abas M, Lund C. Psychological Interventions for Common Mental Disorders for People Living With HIV in Low- and Middle-Income Countries: systematic review. Trop Med Int Health 2015 Jul;20(7):830-839 [FREE Full text] [doi: 10.1111/tmi.12500] [Medline: 25753741]

15. Liu G, Jack H, Piette A, Mangezi W, Machando D, Rwafa C, et al. Mental health training for health workers in Africa: a systematic review. The Lancet Psychiatry 2016 Jan;3(1):65-76. [doi: 10.1016/S2215-0366(15)00379-X]

16. Bass JK, Annan J, McIvor MS, Kaysen D, Griffiths S, Cetinoglu T, et al. Controlled trial of psychotherapy for Congolese survivors of sexual violence. N Engl J Med 2013 Jun 06;368(23):2182-2191. [doi: 10.1056/NEJMoa1211853] [Medline: $\underline{23738545}$

17. Araya R, Flynn T, Rojas G, Fritsch R, Simon G. Cost-effectiveness of a primary care treatment program for depression in low-income women in Santiago, Chile. Am J Psychiatry 2006 Aug;163(8):1379-1387. [doi: 10.1176/ajp.2006.163.8.1379] [Medline: $\underline{16877650}$ ]

18. Rahman A, Malik A, Sikander S, Roberts C, Creed F. Cognitive behaviour therapy-based intervention by community health workers for mothers with depression and their infants in rural Pakistan: a cluster-randomised controlled trial. Lancet 2008 Sep 13;372(9642):902-909 [FREE Full text] [doi: 10.1016/S0140-6736(08)61400-2] [Medline: 18790313]

19. Patel V, Weiss H, Chowdhary N, Naik S, Pednekar S, Chatterjee S, et al. Effectiveness of an intervention led by lay health counsellors for depressive and anxiety disorders in primary care in Goa, India (MANAS): a cluster randomised controlled trial. Lancet 2010 Dec 18;376(9758):2086-2095 [FRE Full text] [doi: 10.1016/S0140-6736(10)61508-5] [Medline: 21159375]

20. Kraemer HC, Wilson GT, Fairburn CG, Agras WS. Mediators and moderators of treatment effects in randomized clinical trials. Arch Gen Psychiatry 2002 Oct;59(10):877-883. [Medline: 12365874]

21. Safer D, Hugo E. Designing a control for a behavioral group therapy. Behavior therapy 2006 Jun;37(2):120-130. [doi: 10.1016/j.beth.2005.06.001]

22. Linnan L, Steckler A. Process evaluation for public health interventions and research. San Francisco, CA: Jossey-Bass; 2002.

23. Garcia-Moreno C, Jansen HAFM, Ellsberg M, Heise L, Watts CH, WHO Multi-country Study on Women's Health and Domestic Violence against Women Study Team. Prevalence of intimate partner violence: findings from the WHO multi-country study on women's health and domestic violence. Lancet 2006 Oct 07;368(9543):1260-1269. [doi: 10.1016/S0140-6736(06)69523-8] [Medline: 17027732]

24. Grekin R, O'Hara M. Prevalence and risk factors of postpartum posttraumatic stress disorder: a meta-analysis. Clin Psychol Rev 2014 Jul;34(5):389-401. [doi: 10.1016/j.cpr.2014.05.003] [Medline: 24952134]

25. Riper H, Andersson G, Hunter S, de Wit J, Berking M, Cuijpers P. Treatment of comorbid alcohol use disorders and depression with cognitive-behavioural therapy and motivational interviewing: a meta-analysis. Addiction 2014 Mar;109(3):394-406 [FREE Full text] [doi: 10.1111/add.12441] [Medline: 24304463]

26. Lambert M, Barley D. Research summary on the therapeutic relationship and psychotherapy outcome. Psychotherapy: Theory, Research, Practice, Training 2001;38(4):357-361. [doi: 10.1037/0033-3204.38.4.357]

27. Ahn H, Wampold B. Where oh where are the specific ingredients? A meta-analysis of component studies in counseling and psychotherapy. Journal of Counseling Psychology 2001;48(3):251-257. [doi: 10.1037/0022-0167.48.3.251]

28. Chan A, Tetzlaff J, Altman D, Laupacis A, Gøtzsche PC, Krleža-Jerić K, et al. SPIRIT 2013 statement: defining standard protocol items for clinical trials. Ann Intern Med 2013 Feb 05;158(3):200-207 [FREE Full text] [doi: 10.7326/0003-4819-158-3-201302050-00583] [Medline: 23295957]

29. Campbell M, Piaggio G, Elbourne D, Altman D, CONSORT Group. Consort 2010 statement: extension to cluster randomised trials. BMJ 2012 Sep 04;345:e5661. [Medline: 22951546] 
30. Delavande A, Menezes C. MICROCON. 2017. Violent Conflicts and Risky Sexual Behavior in Uganda URL: https://pdfs. semanticscholar.org/86fa/b3841f078c73d3fcd61b4664d8eb4f1bf023.pdf [accessed 2017-10-21] [WebCite Cache ID 6uNI1pcpF]

31. Baingana F, Mangen P. Scaling up of mental health and trauma support among war affected communities in northern Uganda. Intervention 2011;9(3):291-303. [doi: 10.1097/WTF.0b013e32834dfcc3]

32. Sheehan D, Lecrubier Y, Sheehan K, Amorim P, Janavs J, Weiller E, et al. The Mini-International Neuropsychiatric Interview (M.I.N.I.): the development and validation of a structured diagnostic psychiatric interview for DSM-IV and ICD-10. J Clin Psychiatry 1998;59 Suppl 20:22-33;quiz 34 [FREE Full text] [Medline: 9881538]

33. Patterson WM, Dohn HH, Bird J, Patterson GA. Evaluation of suicidal patients: the SAD PERSONS scale. Psychosomatics 1983 Apr;24(4):343-45, 348. [doi: 10.1016/S0033-3182(83)73213-5] [Medline: 6867245]

34. World Health Organization. A User's Guide to the Self Reporting Questionnaire (SRQ). Geneva, Switzerland: World Health Organization; 1994:1-84.

35. Nakimuli-Mpungu E, Wamala K, Okello J, Alderman S, Odokonyero R, Musisi S, et al. Developing a culturally sensitive group support intervention for depression among HIV infected and non-infected Ugandan adults: a qualitative study. J Affect Disord 2014 Jul;163:10-17. [doi: 10.1016/j.jad.2014.03.042] [Medline: 24836082]

36. Nakimuli-Mpungu E, Wamala K, Okello J, Alderman S, Odokonyero R, Mojtabai R, et al. Group support psychotherapy for depression treatment in people with HIV/AIDS in northern Uganda: a single-centre randomised controlled trial. Lancet HIV 2015 May;2(5):e190-e199. [doi: 10.1016/S2352-3018(15)00041-7] [Medline: 26423001]

37. Nakimuli-Mpungu E, Wamala K, Okello J, Ndyanabangi S, Kanters S, Mojtabai R, et al. Process Evaluation of a Randomized Controlled Trial of Group Support Psychotherapy for Depression Treatment Among People with HIV/AIDS in Northern Uganda. Community Ment Health J 2017 Nov;53(8):991-1004 [FREE Full text] [doi: 10.1007/s10597-017-0129-4] [Medline: 28317086]

38. Kohrt B, Jordans MJD, Rai S, Shrestha P, Luitel NP, Ramaiya MK, et al. Therapist competence in global mental health: Development of the ENhancing Assessment of Common Therapeutic factors (ENACT) rating scale. Behav Res Ther 2015 Jun;69:11-21 [FREE Full text] [doi: 10.1016/j.brat.2015.03.009] [Medline: 25847276]

39. Alderman S, Lowry B, Nakimuli-Mpungu E. Development of a local function assessment tool: a field-based example from the Peter C Alderman Foundation Psycho-Trauma Clinics in northern Uganda. 2013 Nov 05 Presented at: 141st APHA Annual Meeting; November 2-6, 2013; Boston, MA.

40. Mollica R. Harvard Program in Refugee Trauma. 2004. Measuring trauma, measuring torture: instructions and guidance on the utilization of the Harvard Program in Refugee Trauma's versions of The Hopkins Symptom Checklist-25(HSCL-25) \& The Harvard Trauma Questionnaire (HTQ) URL: http://hprt-cambridge.org/screening/measuring-trauma-measuring-torture/ [accessed 2017-11-27] [WebCite Cache ID 6vGse3e5M]

41. Rosenberg M. Society and the Adolescent Self-Image. Princeton, NJ: Princeton University Press; 1965:1-340.

42. Zimet G, Dahlem N, Zimet S, Farley G. The Multidimensional Scale of Perceived Social Support. Journal of Personality Assessment 1988 Mar;52(1):30-41. [doi: 10.1207/s15327752jpa5201 2]

43. Nyblade L, MacQuarrie K. U.S. Agency for International Development. 2017. Can we measure hiv/aids-related stigma and discrimination? Current knowledge about quantifying stigma in developing countries URL: http://pdf.usaid.gov/pdf_docs/ PNADF263.pdf [accessed 2017-10-21] [WebCite Cache ID 6uNJE8wLM]

44. Saunders JB, Aasland OG, Babor TF, de la Fuente JR, Grant M. Development of the alcohol use disorders identification test (AUDIT): WHO collaborative project on early detection of persons with harmful alcohol consumption - II. Addiction 1993;88(6):791-804. [doi: 10.1111/j.1360-0443.1993.tb02093.x]

45. Carver C, Scheier M, Weintraub J. Assessing coping strategies: a theoretically based approach. J Pers Soc Psychol 1989 Feb;56(2):267-283. [Medline: 2926629]

46. McGuire-Snieckus R, McCabe R, Catty J, Hansson L, Priebe S. A new scale to assess the therapeutic relationship in community mental health care: STAR. Psychol Med 2007 Jan;37(1):85-95. [doi: 10.1017/S0033291706009299] [Medline: 17094819]

47. Purcell DW, Garfein RS, Latka MH, Thiede H, Hudson S, Bonner S, DUIT Study Team. Development, description, and acceptability of a small-group, behavioral intervention to prevent HIV and hepatitis C virus infections among young adult injection drug users. Drug Alcohol Depend 2007 Nov;91 Suppl 1:S73-S80. [doi: 10.1016/j.drugalcdep.2007.03.004] [Medline: $\underline{17466465]}$

48. Hayes R, Moulton L. Cluster randomised trials. In: Chapman \& Hall/CRC Interdisciplinary Statistics Series. Boca Raton, FL: CRC Press Online; 2009:1-398.

49. Singer J, Willett J. Applied longitudinal data analysis: modeling change and event occurrence. Oxford, UK: Oxford University Press; 2003.

50. The R Foundation. 2017. The R Project for Statistical Computing URL: https://www.r-project.org/ [accessed 2017-10-21] [WebCite Cache ID 6uNJfgdxV]

51. Cohen J. Statistical Power Analysis for the Behavioral Sciences, 2nd ed. New York: Routledge; 1988:1-400.

52. Drennan G, Levett A, Swartz L. Hidden dimensions of power and resistance in the translation process: a south African study. Cult Med Psychiatry 1991 Sep;15(3):361-381. [Medline: 1935183] 
53. Braun V, Clarke V. Using thematic analysis in psychology. Qualitative research in psychology 2006;3(2):77-101.

54. Nakimuli-Mpungu E, Wamala K, Okello J, Alderman S, Odokonyero R, Musisi S, et al. Outcomes, feasibility and acceptability of a group support psychotherapeutic intervention for depressed HIV-affected Ugandan adults: a pilot study. J Affect Disord 2014 Sep;166:144-150. [doi: 10.1016/j.jad.2014.05.005] [Medline: 25012423]

55. Lund C. Poverty, inequality and mental health in low- and middle-income countries: time to expand the research and policy agendas. Epidemiol Psychiatr Sci 2015 Feb 23;24(02):97-99. [doi: 10.1017/S2045796015000050]

56. Levi J, Raymond A, Pozniak A, Vernazza P, Kohler P, Hill A. Can the UNAIDS 90-90-90 target be achieved? A systematic analysis of national HIV treatment cascades. BMJ Glob Health 2016;1(2):e000010 [FREE Full text] [doi: 10.1136/bmjgh-2015-000010] [Medline: 28588933]

57. Kaaya SF, Blander J, Antelman G, Cyprian F, Emmons KM, Matsumoto K, et al. Randomized controlled trial evaluating the effect of an interactive group counseling intervention for HIV-positive women on prenatal depression and disclosure of HIV status. AIDS Care 2013;25(7):854-862. [doi: 10.1080/09540121.2013.763891] [Medline: 23383726]

58. Chibanda D, Weiss H, Verhey R, Simms V, Munjoma R, Rusakaniko S, et al. Effect of a Primary Care-Based Psychological Intervention on Symptoms of Common Mental Disorders in Zimbabwe: A Randomized Clinical Trial. JAMA 2016 Dec 27;316(24):2618-2626. [doi: 10.1001/jama.2016.19102] [Medline: 28027368]

59. Ontario Ministry of Health and Long-Term Care. Health equity impact assessment (HEIA). URL: http://www.health.gov.on.ca/ en/pro/programs/heia/tool.aspx [accessed 2017-10-19] [WebCite Cache ID 6uKLOLwHq]

60. Craig P, Dieppe P, Macintyre S, Michie S, Nazareth I, Petticrew M. Developing and evaluating complex interventions: the new Medical Research Council guidance. BMJ 2008 Sep 29:a1655. [doi: 10.1136/bmj.a1655]

\author{
Abbreviations \\ AIDS: acquired immune deficiency syndrome \\ ART: antiretroviral therapy \\ GHE: group HIV education \\ GSP: group support psychotherapy \\ ITT: intention-to-treat \\ LHW: lay health worker \\ MINI: Mini International Neuropsychiatric Interview \\ PHC: primary care health center \\ PLWH: person living with HIV \\ SEEK-GSP: Social, Emotional, and Economic empowerment through Knowledge of Group Support Psychotherapy \\ TASO: The AIDS Support Organization \\ WHO: World Health Organization
}

Edited by G Eysenbach; submitted 09.09.17; peer-reviewed by S Abdulrahman; comments to author 10.10.17; revised version received
23.10.17; accepted 29.10.17; published 11.12.17
Please cite as:
Nakimuli-Mpungu E, Musisi S, Wamala K, Okello J, Ndyanabangi S, Mojtabai R, Nachega J, Harari O, Mills E
The Effect of Group Support Psychotherapy Delivered by Trained Lay Health Workers for Depression Treatment Among People with
HIV in Uganda: Protocol of a Pragmatic, Cluster Randomized Trial
JMIR Res Protoc 2017;6(12):e250
URL: http://www.researchprotocols.org/2017/12/e250/
doi: $\underline{10.2196 / \text { resprot.8925 }}$
PMID: $\underline{29229589}$

(CEtheldreda Nakimuli-Mpungu, Seggane Musisi, Kizito Wamala, James Okello, Sheila Ndyanabangi, Ramin Mojtabai, Jean Nachega, Ofir Harari, Edward Mills. Originally published in JMIR Research Protocols (http://www.researchprotocols.org), 11.12.2017. This is an open-access article distributed under the terms of the Creative Commons Attribution License (https://creativecommons.org/licenses/by/4.0/), which permits unrestricted use, distribution, and reproduction in any medium, provided the original work, first published in JMIR Research Protocols, is properly cited. The complete bibliographic information, a link to the original publication on http://www.researchprotocols.org, as well as this copyright and license information must be included. 